Journal of Algebra Combinatorics Discrete Structures and Applications

\title{
Some results on relative dual Baer property
}

\author{
Tayyebeh Amouzegar, Rachid Tribak
}

\begin{abstract}
Let $R$ be a ring. In this article, we introduce and study relative dual Baer property. We characterize $R$-modules $M$ which are $R_{R}$-dual Baer, where $R$ is a commutative principal ideal domain. It is shown that over a right noetherian right hereditary ring $R$, an $R$-module $M$ is $N$-dual Baer for all $R$-modules $N$ if and only if $M$ is an injective $R$-module. It is also shown that for $R$-modules $M_{1}, M_{2}, \ldots, M_{n}$ such that $M_{i}$ is $M_{j}$-projective for all $i>j \in\{1,2, \ldots, n\}$, an $R$-module $N$ is $\bigoplus_{i=1}^{n} M_{i}$-dual Baer if and only if $N$ is $M_{i}$-dual Baer for all $i \in\{1,2, \ldots, n\}$. We prove that an $R$-module $M$ is dual Baer if and only if $S=\operatorname{End}_{R}(M)$ is a Baer ring and $I M=r_{M}\left(l_{S}(I M)\right)$ for every right ideal $I$ of $S$.
\end{abstract}

2010 MSC: 16D10, 16D 80

Keywords: Baer rings, Dual Baer modules, Relative dual Baer property, Homomorphisms of modules

\section{Introduction}

Throughout this paper, $R$ will denote an associative ring with identity, and all modules are unitary right $R$-modules. Let $M$ be an $R$-module. We will use the notation $N \ll M$ to indicate that $N$ is small in $M$ (i.e., $L+N \neq M$ for every proper submodule $L$ of $M)$. By $E(M)$ and $E n d_{R}(M)$, we denote the injective hull of $M$ and the endomorphism $\operatorname{ring}$ of $M$, respectively. By $\mathbb{Q}, \mathbb{Z}$, and $\mathbb{N}$ we denote the set of rational numbers, integers and natural numbers, respectively. For a prime number $p, \mathbb{Z}\left(p^{\infty}\right)$ denotes the Prüfer $p$-group.

The concept of Baer rings was first introduced in [6] by Kaplansky. Since then, many authors have studied this kind of rings (see, e.g., [2] and [3]). A ring $R$ is called Baer if the right annihilator of any nonempty subset of $R$ is generated by an idempotent. In 2004, Rizvi and Roman extended the notion of Baer rings to a module theoretic version [10]. According to [10], a module $M$ is called a Baer module if for every left ideal $I$ of $\operatorname{End}_{R}(M), \cap_{\phi \in I} \operatorname{Ker} \phi$ is a direct summand of $M$. This notion was recently dualized by Keskin Tütüncü-Tribak in [14]. A module $M$ is said to be dual Baer if for every right ideal

Tayyebeh Amouzegar; Department of Mathematics, Quchan University of Advanced Technology, Quchan, Iran (email: t.amoozegar@yahoo.com).

Rachid Tribak (Corresponding Author); Centre Régional des Métiers de l'Education et de la Formation (CRMEFTTH)-Tanger, Avenue My Abdelaziz, Souani, B.P. 3117, Tangier, Morocco (email: tribak12@yahoo.com). 
$I$ of $S=\operatorname{End}_{R}(M), \sum_{\phi \in I} \operatorname{Im} \phi$ is a direct summand of $M$. Equivalently, for every nonempty subset $A$ of $S, \sum_{\phi \in A} \operatorname{Im} \phi$ is a direct summand of $M$ (see [14, Theorem 2.1]).

A module $M$ is said to be Rickart if for any $\varphi \in \operatorname{End}_{R}(M), \operatorname{Ker} \varphi$ is a direct summand of $M$ (see [7]). The notion of dual Rickart modules was studied recently in [8] by Lee-Rizvi-Roman. A module $M$ is said to be dual Rickart if for every $\varphi \in \operatorname{End}_{R}(M), \operatorname{Im} \varphi$ is a direct summand of $M$. In [8], it was introduced the notion of relative dual Rickart property which was used in the study of direct sums of dual Rickart modules. Let $N$ be an $R$-module. An $R$-module $M$ is called $N$-dual Rickart if for every homomorphism $\varphi: M \rightarrow N, \operatorname{Im} \varphi$ is a direct summand of $N$ (see [8]). Similarly, we introduce in this paper the concept of relative dual Baer property. A module $M$ is called $N$-dual Baer if for every subset $A$ of $\operatorname{Hom}_{R}(M, N)$, $\sum_{f \in A} \operatorname{Im} f$ is a direct summand of $N$. It is clear that if $M$ is $N$-dual Baer, then $M$ is $N$-dual Rickart.

We determine the structure of modules $M$ which are $R_{R}$-dual Baer for a commutative principal ideal domain $R$ (Proposition 2.7). Then we show that for an $R$-module $M, R_{R}$ is $M$-dual Baer if and only if $M$ is a semisimple module (Proposition 2.9). It is shown that over a right noetherian right hereditary ring $R$, an $R$-module $M$ is $N$-dual Baer for all $R$-modules $N$ if and only if $M$ is an injective $R$-module (Corollary 2.17). We prove that if $\left\{M_{i}\right\}_{I}$ is a family of $R$-modules, then for each $j \in I, \bigoplus_{i \in I} M_{i}$ is $M_{j}$-dual Baer if and only if $M_{i}$ is $M_{j}$-dual Baer for all $i \in I$ (Corollary 2.24). It is also shown that for $R$-modules $M_{1}$, $M_{2}, \ldots, M_{n}$ such that $M_{i}$ is $M_{j}$-projective for all $i>j \in\{1,2, \ldots, n\}$, an $R$-module $N$ is $\bigoplus_{i=1}^{n} M_{i}$-dual Baer if and only if $N$ is $M_{i}$-dual Baer for all $i \in\{1,2, \ldots, n\}$ (Theorem 2.25). We conclude this paper by showing that an $R$-module $M$ is dual Baer if and only if $S=\operatorname{End}_{R}(M)$ is a Baer ring and $I M=r_{M}\left(l_{S}(I)\right)$ for every right ideal $I$ of $S$, where $l_{S}(I)=\{\varphi \in S \mid \varphi I=0\}, r_{M}\left(l_{S}(I)\right)=\left\{m \in M \mid l_{S}(I) m=0\right\}$ and $I M=\sum_{f \in I} \operatorname{Im} f$ (Theorem 2.31).

\section{Main results}

Definition 2.1. Let $N$ be an $R$-module. An $R$-module $M$ is called $N$-dual Baer if, for every subset $A$ of $\operatorname{Hom}_{R}(M, N), \sum_{f \in A} \operatorname{Im} f$ is a direct summand of $N$.

Obviously, an $R$-module $M$ is dual Baer if and only if $M$ is $M$-dual Baer.

Example 2.2. (1) Let $N$ be a semisimple $R$-module. Then for every $R$-module $M, M$ is $N$-dual Baer.

(2) If $M$ and $N$ are $R$-modules such that $\operatorname{Hom}_{R}(M, N)=0$, then $M$ is $N$-dual Baer. It follows that for any couple of different maximal ideals $\mathfrak{m}_{1}$ and $\mathfrak{m}_{2}$ of a commutative noetherian ring $R, E\left(R / \mathfrak{m}_{1}\right)$ is $E\left(R / \mathfrak{m}_{2}\right)$-dual Baer (see [12, Proposition 4.21]).

(3) Let $p$ be a prime number. Note that $\mathbb{Z} / p \mathbb{Z}$ and $\mathbb{Z}\left(p^{\infty}\right)$ are dual Baer $\mathbb{Z}$-modules. On the other hand, it is clear that $\mathbb{Z}\left(p^{\infty}\right)$ is $\mathbb{Z} / p \mathbb{Z}$-dual Baer but $\mathbb{Z} / p \mathbb{Z}$ is not $\mathbb{Z}\left(p^{\infty}\right)$-dual Baer.

Recall that a module $M$ is said to have the strong summand sum property, denoted briefly by $S S S P$, if the sum of any family of direct summands of $M$ is a direct summand of $M$.

Following [8, Definition 2.14], a module $M$ is called $N$-d-Rickart if, for every homomorphism $\varphi$ : $M \rightarrow N, \operatorname{Im} \varphi$ is a direct summand of $N$.

Proposition 2.3. Let $M$ and $N$ be two R-modules. If $M$ is $N$-dual Baer, then $M$ is $N$-d-Rickart. The converse holds when $N$ has the SSSP.

Proof. This follows from the definitions of " $M$ is $N$-d-Rickart" and " $M$ is $N$-dual Baer". 2.3

The next example shows that the assumption " $N$ has the $S S S P$ " is not superfluous in Proposition

Example 2.4. Let $R$ be a von Neumann regular ring which is not semisimple (e.g., $R=\prod_{i=1}^{\infty} \mathbb{Z} / 2 \mathbb{Z}$ ). By [8, Proposition 2.26], the $R$-module $R_{R}$ does not have the $S S S P$. On the other hand, $R_{R}$ is $R_{R}$-d-Rickart, but it is not $R_{R}$-dual Baer (see [14, Corollary 2.9] and [8, Remark 2.2]). 
Proposition 2.5. Let $N$ be an indecomposable $R$-module. Then the following conditions are equivalent for an $R$-module $M$.

(i) $M$ is $N$-dual Baer;

(ii) $M$ is $N$-d-Rickart;

(iii) Every nonzero $\varphi \in \operatorname{Hom}_{R}(M, N)$ is an epimorphism.

Proof. (i) $\Rightarrow$ (ii) and (iii) $\Rightarrow$ (i) are clear.

(ii) $\Rightarrow$ (iii) Let $0 \neq \varphi \in \operatorname{Hom}_{R}(M, N)$. By assumption, $\operatorname{Im} \varphi$ is a direct summand of $N$. But $N$ is indecomposable. Then $\operatorname{Im} \varphi=N$. This completes the proof.

Proposition 2.6. Let $M$ and $N$ be modules such that $\operatorname{Hom}_{R}(M, N) \neq 0($ e.g., $N$ is $M$-generated $)$. Then the following conditions are equivalent:

(i) $M$ is $N$-dual Baer and $N$ is indecomposable;

(ii) Every nonzero homomorphism $\varphi \in \operatorname{Hom}_{R}(M, N)$ is an epimorphism.

Proof. $\quad$ (i) $\Rightarrow$ (ii) This follows from Proposition 2.5.

(ii) $\Rightarrow$ (i) It is clear that $M$ is $N$-dual Baer. Now let $K$ be a nonzero direct summand of $N$. Let $K^{\prime}$ be a submodule of $N$ such that $N=K \oplus K^{\prime}$. Since $\operatorname{Hom}_{R}(M, N) \neq 0$, there exists a nonzero homomorphism $\varphi \in \operatorname{Hom}_{R}(M, N)$. Let $\pi^{\prime}: N \rightarrow K^{\prime}$ be the projection map and let $i^{\prime}: K^{\prime} \rightarrow N$ be the inclusion map. Then $i^{\prime} \pi^{\prime} \varphi \in \operatorname{Hom}_{R}(M, N)$. Assume that $i^{\prime} \pi^{\prime} \varphi \neq 0$. By hypothesis, $\operatorname{Im} i^{\prime} \pi^{\prime} \varphi=N$. So $K^{\prime}=N$. Thus $K=0$, a contradiction. Therefore $i^{\prime} \pi^{\prime} \varphi=0$. Hence $K^{\prime}=0$ and $K=N$. It follows that $N$ is indecomposable.

The following result describes the structure of $R$-modules which are $R_{R}$-dual Baer, where $R$ is a commutative principal ideal domain which is not a field.

Proposition 2.7. Let $R$ be a commutative principal ideal domain which is not a field. Then the following conditions are equivalent for an $R$-module $M$ :

(i) $M$ is $R_{R}$-dual Baer;

(ii) $M$ is $R_{R}$-d-Rickart;

(iii) $M$ has no nonzero cyclic torsion-free direct summands;

(iv) $\operatorname{Hom}_{R}\left(M, R_{R}\right)=0$.

Proof. (i) $\Rightarrow$ (ii) This is clear.

(ii) $\Rightarrow$ (iii) Assume that $M$ has an element $x$ such that $x R$ is a direct summand of $M$ and $R_{R} \cong x R$. Let $\pi: M \rightarrow x R$ be the projection map and let $f: x R \rightarrow R_{R}$ be an isomorphism. Then $f \pi: M \rightarrow R_{R}$ is an epimorphism. Let $\alpha$ be a nonzero element of $R$ which is not invertible. Consider the homomorphism $g: R_{R} \rightarrow R_{R}$ defined by $g(r)=\alpha r$ for all $r \in R$. Then $g f \pi \in H_{o m}\left(M, R_{R}\right)$ and $\operatorname{Im} g f \pi=\alpha R$. It is clear that $\alpha R \neq 0$ and $\alpha R \neq R$. Thus $\alpha R$ is not a direct summand of $R$. So $M$ is not $R_{R}$-d-Rickart, a contradiction.

(iii) $\Rightarrow$ (iv) Assume that $\operatorname{Hom}_{R}\left(M, R_{R}\right) \neq 0$. So there exists a nonzero homomorphism $f: M \rightarrow R_{R}$. Thus $\operatorname{Im} f=a R$ for some nonzero $a \in R$ since $R$ is a principal ideal domain. Then $M / \operatorname{Ker} f \cong a R \cong R_{R}$ is a projective $R$-module. It follows that $\operatorname{Ker} f$ is a direct summand of $M$. Let $Y$ be a submodule of $M$ such that $M=\operatorname{Ker} f \oplus Y$. Therefore $Y \cong R_{R}$. This contradicts our assumption. Hence $\operatorname{Hom}_{R}\left(M, R_{R}\right)=0$.

(iv) $\Rightarrow$ (i) This is immediate.

Example 2.8. Consider a $\mathbb{Z}$-module $M=\mathbb{Q}^{(I)} \oplus T$, where $T$ is a torsion $\mathbb{Z}$-module and $I$ is an index set. Suppose that $M$ is not $\mathbb{Z}$-dual Baer. By Proposition 2.7, there exists a cyclic submodule $L$ of $M$ such that $L \cong \mathbb{Z}$ and $L$ is a direct summand of $M$. Let $N$ be a submodule of $M$ such that $M=L \oplus N$. 
Since $T$ is the torsion submodule of $M$, we have $T \subseteq N$. Hence $T$ is a direct summand of $N$. Let $K$ be a submodule of $N$ such that $N=K \oplus T$. Thus $M=\bar{L} \oplus K \oplus T$. Therefore $L \oplus K \cong \mathbb{Q}^{(I)}$. So $L$ is injective, a contradiction. It follows that $M$ is $\mathbb{Z}$-dual Baer. On the other hand, note that if $T \cong \mathbb{Z}\left(2^{\infty}\right) \oplus \mathbb{Z} / 8 \mathbb{Z}$, then $M$ is not a dual Baer module (see [14, Corollary 3.5].

In Proposition 2.7, we studied when an $R$-module $M$ is $R_{R}$-dual Baer. Next, we investigate when $R_{R}$ is $M$-dual Baer for an $R$-module $M$.

Proposition 2.9. The following conditions are equivalent for an $R$-module $M$ :

(i) The R-module $R_{R}$ is $M$-dual Baer;

(ii) $M$ is a semisimple module.

Proof. (i) $\Rightarrow$ (ii) Let $x \in M$. Consider the $R$-homomorphism $\varphi: R \rightarrow M$ defined by $\varphi(r)=x r$ for all $r \in R$. Then $\operatorname{Im} \varphi=x R$. Since $R_{R}$ is $M$-dual Baer, it follows that for any submodule $L$ of $M$, $L=\sum_{x \in L} x R$ is a direct summand of $M$. Therefore $M$ is semisimple.

(ii) $\Rightarrow$ (i) is obvious.

Corollary 2.10. The following conditions are equivalent for a ring $R$ :

(i) The R-module $R_{R}$ is dual Baer;

(ii) The $R$-module $R_{R}$ is $E(R)$-dual Baer;

(iii) $R$ is a semisimple ring.

Proof. $\quad($ i) $\Leftrightarrow$ (iii) By [14, Corollary 2.9].

(ii) $\Leftrightarrow$ (iii) This follows from Proposition 2.9.

Remark 2.11. If $K$ is a submodule of an $R$-module $M$ such that $K$ is $M$-dual Baer, then $K$ is a direct summand of $M$. In particular, if the $R$-module $M$ is $E(M)$-dual Baer, then $M$ is an injective module. Baer

The next example shows that even if a module $M$ is injective, the module $M$ need not be $M$-dual

Example 2.12. Let $R$ be a self injective ring which is not semisimple (e.g., $R=\prod_{n=1}^{\infty} \mathbb{Z} / 2 \mathbb{Z}$ ). Then $E\left(R_{R}\right)=R_{R}$. By [14, Corollary 2.9], the $R$-module $R_{R}$ is not $R_{R}$-dual Baer.

Next, we will be concerned with the modules $M$ which are $N$-dual Baer for all modules $N$. We begin with the following proposition which provides a class of rings $R$ whose semisimple modules are $N$-dual Baer for any $R$-module $N$.

Proposition 2.13. Let $R$ be a right noetherian right $V$-ring and let $M$ be a semisimple $R$-module. Then $M$ is $N$-dual Baer for every $R$-module $N$.

Proof. Let $N$ be an $R$-module. It is clear that for any $\varphi \in \operatorname{Hom}_{R}(M, N), \operatorname{Im} \varphi$ is semisimple. Let $A$ be a subset of $\operatorname{Hom}_{R}(M, N)$. Then $\sum_{f \in A} \operatorname{Im} f$ is a semisimple submodule of $N$. Since $R$ is a right noetherian right V-ring, $\sum_{f \in A} \operatorname{Im} f$ is injective by [4, Proposition 1]. Therefore $\sum_{f \in A} \operatorname{Im} f$ is a direct summand of $N$. So $M$ is $N$-dual Baer.

The next example shows that the condition " $R$ is a right noetherian ring" in the hypothesis of Proposition 2.13 is not superfluous.

Example 2.14. Let $F$ be a field and let $R=\prod_{n \in \mathbb{N}} F_{n}$ such that $F_{n}=F$ for all $n \in \mathbb{N}$. Then $R$ is a commutative V-ring which is not noetherian. Note that $\operatorname{Soc}(R)=\oplus_{n \in \mathbb{N}} F_{n}$ is an essential proper ideal of $R$. In particular, $\operatorname{Soc}(R)$ is not a direct summand of $R$. So $\operatorname{Soc}(R)$ is not $R_{R}$-dual Baer. 
Following [13], a module $M$ is called noncosingular if for every nonzero module $N$ and every nonzero homomorphism $f: M \rightarrow N, \operatorname{Im} f$ is not a small submodule of $N$.

Proposition 2.15. Let $M$ be a module. Assume that $M$ is $N$-dual Baer for every R-module $N$. Then every factor module of $M$ is injective. In particular, $M$ is a noncosingular module.

Proof. Let $L$ be a submodule of $M$. Let $\pi: M \rightarrow M / L$ be the natural epimorphism and let $\mu$ : $M / L \rightarrow E(M / L)$ be the inclusion map. Then $\mu \pi \in H \operatorname{Hom}_{R}(M, E(M / L))$ and $\operatorname{Im} \mu \pi=M / L$. Since $M$ is $E(M / L)$-dual Baer, $M / L$ is a direct summand of $E(M / L)$. So $M / L$ is injective. This completes the proof.

Proposition 2.16. Let $R$ be a right noetherian ring. Then the following conditions are equivalent for an $R$-module $M$ :

(i) $M$ is $N$-dual Baer for all R-modules $N$;

(ii) Every factor module of $M$ is an injective $R$-module.

Proof. $\quad$ (i) $\Rightarrow$ (ii) By Proposition 2.15.

(ii) $\Rightarrow$ (i) Let $N$ be an $R$-module. It is clear that $\operatorname{Im} \varphi$ is injective for every $\varphi \in \operatorname{Hom}_{R}(M, N)$. Since the ring $R$ is right noetherian, $\sum_{f \in A} \operatorname{Im} f$ is injective for every subset $A$ of $\operatorname{Hom}_{R}(M, N)$ by $[1$, Proposition 18.13]. Therefore $\sum_{f \in A} \operatorname{Im} f$ is a direct summand of $N$. This proves the proposition.

Recall that a ring $R$ is called right hereditary if each of its right ideals is projective. It is well known that a ring $R$ is right hereditary if and only if every factor module of an injective right $R$-module is injective (see, for example $[16,39.16])$. The next result is a direct consequence of Proposition 2.16. It determines the structure of $R$-modules $M$ which are $N$-dual Baer for all $R$-modules $N$, where $R$ is a right noetherian right hereditary ring.

Corollary 2.17. Let $R$ be a right noetherian right hereditary ring (e.g., $R$ is a Dedekind domain). Then the following conditions are equivalent for an $R$-module $M$ :

(i) $M$ is $N$-dual Baer for any $R$-module $N$;

(ii) $M$ is an injective $R$-module.

Example 2.18. Let $M$ be a $\mathbb{Z}$-module. It is easily seen from Corollary 2.17 that $M$ is $N$-dual Baer for any $\mathbb{Z}$-module $N$ if and only if $M$ is a direct sum of $\mathbb{Z}$-modules each isomorphic to the additive group of rational numbers $\mathbb{Q}$ or to $\mathbb{Z}\left(p^{\infty}\right)$ (for various primes $p$ ).

Combining Corollary 2.17 and [8, Corollary 2.30], we obtain the following result.

Corollary 2.19. The following conditions are equivalent for a ring $R$ :

(i) Every injective $R$-module is dual Baer;

(ii) Every injective module is $N$-dual Baer for every $R$-module $N$;

(iii) $R$ is a right noetherian right hereditary ring.

The next characterization extends [14, Corollary 2.5].

Theorem 2.20. Let $M$ and $N$ be two R-modules. Then $M$ is $N$-dual Baer if and only if for any direct summand $M^{\prime}$ of $M$ and any submodule $N^{\prime}$ of $N, M^{\prime}$ is $N^{\prime}$-dual Baer.

Proof. Let $M^{\prime}=e M$ for some $e^{2}=e \in \operatorname{End}_{R}(M)$ and let $N^{\prime}$ be a submodule of $N$. Let $\left\{\varphi_{i}\right\}_{I}$ be a family of homomorphisms in $\operatorname{Hom}_{R}\left(M^{\prime}, N^{\prime}\right)$. Since $\varphi_{i} e(M)=\varphi_{i}\left(M^{\prime}\right) \subseteq N^{\prime} \subseteq N$ for every $i \in I$ and $M$ is $N$-dual Baer, $\sum_{i \in I} \varphi_{i} e(M)$ is a direct summand of $N$. Therefore $\sum_{i \in I} \varphi_{i}\left(\bar{M}^{\prime}\right)$ is a direct summand of $N^{\prime}$. It follows that $M^{\prime}$ is $N^{\prime}$-dual Baer. The converse is obvious. 
Corollary 2.21. The following conditions are equivalent for a module $M$ :

(i) $M$ is a dual Baer module;

(ii) For any direct summand $K$ of $M$ and any submodule $N$ of $M, K$ is $N$-dual Baer.

From [14, Example 3.1 and Theorem 3.4], it follows that a direct sum of dual Baer modules is not dual Baer, in general. Next, we focus on when a direct sum of $N$-dual Baer modules is also $N$-dual Baer for some module $N$.

Proposition 2.22. Let $N$ be a module having the SSSP and let $\left\{M_{i}\right\}_{I}$ be a family of modules. Then $\bigoplus_{i \in I} M_{i}$ is $N$-dual Baer if and only if $M_{i}$ is $N$-dual Baer for all $i \in I$.

Proof. Suppose that $\bigoplus_{i \in I} M_{i}$ is $N$-dual Baer. By Theorem 2.20, $M_{i}$ is $N$-dual Baer for all $i \in I$. Conversely, assume that $M_{i}$ is $N$-dual Baer for all $i \in I$. Let $\left\{\varphi_{\lambda}\right\}_{\Lambda}$ be a family of homomorphisms in $\operatorname{Hom}_{R}\left(\bigoplus_{i \in I} M_{i}, N\right)$. For each $i \in I$, let $\mu_{i}: M_{i} \rightarrow \bigoplus_{i \in I} M_{i}$ denote the inclusion map. Then for every $i \in I$ and every $\lambda \in \Lambda, \varphi_{\lambda} \mu_{i} \in \operatorname{Hom}_{R}\left(M_{i}, N\right)$. Since $M_{i}$ is $N$-dual Baer for every $i \in I$, it follows that $\operatorname{Im}\left(\varphi_{\lambda} \mu_{i}\right)$ is a direct summand of $N$ for every $(i, \lambda) \in I \times \Lambda$. Note that for each $\lambda \in \Lambda$, $\operatorname{Im} \varphi_{\lambda}=\sum_{i \in I} \operatorname{Im}\left(\varphi_{\lambda} \mu_{i}\right)$. As $N$ has the $S S S P, \sum_{\lambda \in \Lambda} \operatorname{Im} \varphi_{\lambda}=\sum_{\lambda \in \Lambda} \sum_{i \in I} \operatorname{Im}\left(\varphi_{\lambda} \mu_{i}\right)$ is a direct summand of $N$. Therefore $\bigoplus_{i \in I} M_{i}$ is $N$-dual Baer.

The following result is taken from [14, Theorem 2.1].

Theorem 2.23. The following conditions are equivalent for a module $M$ and $S=\operatorname{End}_{R}(M)$ :

(i) $M$ is a dual Baer module;

(ii) For every nonempty subset $A$ of $S, \sum_{f \in A} \operatorname{Im} f=e(M)$ for some idempotent $e \in S$;

(iii) $M$ has the SSSP and for every $\varphi: M \rightarrow M, \operatorname{Im} \varphi$ is a direct summand of $M$.

Corollary 2.24. Let $\left\{M_{i}\right\}_{I}$ be a family of modules and let $j \in I$. Then $\bigoplus_{i \in I} M_{i}$ is $M_{j}$-dual Baer if and only if $M_{i}$ is $M_{j}$-dual Baer for all $i \in I$.

Proof. The necessity follows from Theorem 2.20. Conversely, by assumption, we have $M_{j}$ is $M_{j}$-dual Baer. Then $M_{j}$ is a dual Baer module. By Theorem 2.23, $M_{j}$ has the SSSP. Applying Proposition 2.22, $\bigoplus_{i \in I} M_{i}$ is $M_{j}$-dual Baer.

In the following result, we present conditions under which a module $N$ is $\bigoplus_{i=1}^{n} M_{i}$-dual Baer for some modules $M_{i}(1 \leq i \leq n)$.

Theorem 2.25. Let $M_{1}, \ldots, M_{n}$ be $R$-modules, where $n \in \mathbb{N}$. Assume that $M_{i}$ is $M_{j}$-projective for all $i>j \in\{1,2, \ldots, n\}$. Then for any $R$-module $N, N$ is $\bigoplus_{i=1}^{n} M_{i}$-dual Baer if and only if $N$ is $M_{i}$-dual Baer for all $i \in\{1,2, \ldots, n\}$.

Proof. The necessity follows from Theorem 2.20. Conversely, suppose that $N$ is $M_{i}$-dual Baer for all $i \in\{1,2, \ldots, n\}$. We will show that $N$ is $\bigoplus_{i=1}^{n} M_{i}$-dual Baer. By induction on $n$ and taking into account [9, Proposition 4.33], it is sufficient to prove this for the case $n=2$. Assume that $N$ is $M_{i}$-dual Baer for $i=1,2$ and $M_{2}$ is $M_{1}$-projective. Let $\left\{\phi_{\lambda}\right\}_{\Lambda}$ be a family of homomorphisms in $\operatorname{Hom}_{R}\left(N, M_{1} \oplus M_{2}\right)$. Let $\pi_{2}: M_{1} \oplus M_{2} \rightarrow M_{2}$ be the projection of $M_{1} \oplus M_{2}$ on $M_{2}$ along $M_{1}$. We want to prove that $\sum_{\lambda \in \Lambda} \operatorname{Im} \phi_{\lambda}$ is a direct summand of $M_{1} \oplus M_{2}$. Since $N$ is $M_{2}$-dual Baer, $\sum_{\lambda \in \Lambda} \pi_{2} \phi_{\lambda}(N)$ is a direct summand of $M_{2}$. So $\sum_{\lambda \in \Lambda} \pi_{2} \phi_{\lambda}(N)$ is $M_{1}$-projective by [9, Proposition 4.32]. As $M_{1}+\left(\sum_{\lambda \in \Lambda} \operatorname{Im} \phi_{\lambda}\right)=$ $M_{1} \oplus\left(\sum_{\lambda \in \Lambda} \pi_{2} \phi_{\lambda}(N)\right)$ is a direct summand of $M_{1} \oplus M_{2}$, there exists a submodule $L \leq \sum_{\lambda \in \Lambda} \operatorname{Im} \phi_{\lambda}$ such that $M_{1}+\left(\sum_{\lambda \in \Lambda} \operatorname{Im} \phi_{\lambda}\right)=M_{1} \oplus L$ by [9, Lemma 4.47]. Thus $\sum_{\lambda \in \Lambda} \operatorname{Im} \phi_{\lambda}=\left(M_{1} \cap\left(\sum_{\lambda \in \Lambda} \operatorname{Im} \phi_{\lambda}\right)\right) \oplus L$ by modularity. It is easily seen that $\sum_{\lambda \in \Lambda} \pi_{2} \phi_{\lambda}(N)$ is a direct summand of $M_{2}$. Let $K_{2}$ be a submodule of $M_{2}$ such that $M_{2}=K_{2} \oplus\left(\sum_{\lambda \in \Lambda} \pi_{2} \phi_{\lambda}(N)\right)$. Therefore $M_{1} \oplus M_{2}=M_{1} \oplus L \oplus K_{2}$. Let $\pi_{1}: M_{1} \oplus(L \oplus K) \rightarrow M_{1}$ 
be the projection of $M_{1} \oplus M_{2}$ on $M_{1}$ along $L \oplus K$. Then $\pi_{1} \phi_{\lambda} \in \operatorname{Hom}_{R}\left(N, M_{1}\right)$ for every $\lambda \in \Lambda$. Moreover, we have

$$
\sum_{\lambda \in \Lambda} \pi_{1} \phi_{\lambda}(N)=\pi_{1}\left(\sum_{\lambda \in \Lambda} \operatorname{Im} \phi_{\lambda}\right)=\left(\left(\sum_{\lambda \in \Lambda} \operatorname{Im} \phi_{\lambda}\right)+(L \oplus K)\right) \cap M_{1} .
$$

But $\sum_{\lambda \in \Lambda} \operatorname{Im} \phi_{\lambda}=\left(M_{1} \cap\left(\sum_{\lambda \in \Lambda} \operatorname{Im} \phi_{\lambda}\right)\right) \oplus L$. Then,

$$
\sum_{\lambda \in \Lambda} \pi_{1} \phi_{\lambda}(N)=\left(\left(M_{1} \cap\left(\sum_{\lambda \in \Lambda} \operatorname{Im} \phi_{\lambda}\right)\right) \oplus L \oplus K\right) \cap M_{1}=M_{1} \cap\left(\sum_{\lambda \in \Lambda} \operatorname{Im} \phi_{\lambda}\right) .
$$

Since $N$ is $M_{1}$-dual Baer, $\sum_{\lambda \in \Lambda} \pi_{1} \phi_{\lambda}(N)=M_{1} \cap\left(\sum_{\lambda \in \Lambda} \operatorname{Im} \phi_{\lambda}\right)$ is a direct summand of $M_{1}$. It follows that $\left(M_{1} \cap\left(\sum_{\lambda \in \Lambda} \operatorname{Im} \phi_{\lambda}\right)\right) \oplus L$ is a direct summand of $M_{1} \oplus L \oplus K_{2}$. So $\sum_{\lambda \in \Lambda} \operatorname{Im} \phi_{\lambda}$ is a direct summand of $M_{1} \oplus M_{2}$. Consequently, $N$ is $M_{1} \oplus M_{2}$-dual Baer. This completes the proof.

Corollary 2.26. Let $M_{1}, \ldots, M_{n}$ be $R$-modules, where $n \in \mathbb{N}$. Assume that $M_{i}$ is $M_{j}$-projective for all $i>j \in\{1,2, \ldots, n\}$. Then $M=\bigoplus_{i=1}^{n} M_{i}$ is a dual Baer module if and only if $M_{i}$ is $M_{j}$-dual Baer for all $i, j \in\{1,2, \ldots, n\}$.

Proof. The necessity follows from Theorem 2.20. Conversely, suppose that $M_{i}$ is $M_{j}$-dual Baer for all $i, j \in\{1,2, \ldots, n\}$. By Corollary 2.24, $M$ is $M_{j}$-dual Baer for all $j \in\{1,2, \ldots, n\}$. Since $M_{i}$ is $M_{j}$-projective for all $i>j \in\{1,2, \ldots, n\}, M$ is $\bigoplus_{i=1}^{n} M_{i}$-dual Baer by Theorem 2.25. Thus $M$ is a dual Baer module.

Note that the sufficiency in Corollary 2.26 can be proved by using [14, Theorem 3.10].

Following [8, Definition 5.7], a module $M$ is called $N-D_{2}$ (or relatively $D_{2}$ to $N$ ) if for any submodule $M^{\prime}$ of $M, M / M^{\prime}$ is isomorphic to a direct summand of $N$ implies that $M^{\prime}$ is a direct summand of $M$.

Proposition 2.27. Let $M_{1}, \ldots, M_{n}$ be R-modules, where $n \in \mathbb{N}$. Assume that $M_{i}$ is $M_{j}-D_{2}$ for all $i, j \in\{1,2, \ldots, n\}$. Then $\bigoplus_{i=1}^{n} M_{i}$ is a dual Baer module if and only if $M_{i}$ is $M_{j}$-dual Baer for all $i, j \in\{1,2, \ldots, n\}$ and $M$ has the $S S S P$.

Proof. $(\Rightarrow)$ By [8, Theorem 5.11], $M_{i}$ is $M_{j}$-d-Rickart for all $i, j \in\{1,2, \ldots, n\}$. Note that $M_{i}$ has the $S S S P$ for every $i \in\{1,2, \ldots, n\}$ (see Theorem 2.23). Applying Proposition 2.3, it follows that $M_{i}$ is $M_{j}$-dual Baer for all $i, j \in\{1,2, \ldots, n\}$.

$(\Leftarrow)$ This follows easily from [8, Theorem 5.11], Proposition 2.3 and Theorem 2.23.

Theorem 2.28. Let $M=\bigoplus_{i \in I} M_{i}$ be the direct sum of fully invariant submodules $M_{i}$. Then $M$ is a dual Baer module if and only if $M_{i}$ is a dual Baer module for all $i \in I$.

Proof. The necessity follows from [14, Corollary 2.5]. Conversely, let $S=\operatorname{End}_{R}(M)$ and let $\left\{\varphi_{\lambda}\right\}_{\Lambda}$ be a family of homomorphisms in $S$. For each $i \in I$, let $\pi_{i}: M \rightarrow M_{i}$ be the projection map and let $\mu_{i}: M_{i} \rightarrow M$ be the inclusion map. Note that for each $\lambda \in \Lambda, \varphi_{\lambda}(M)=\sum_{i \in I} \varphi_{\lambda} \mu_{i}\left(M_{i}\right)$. Since each $M_{i}$ $(i \in I)$ is fully invariant in $M$, it follows that $\varphi_{\lambda}(M)=\sum_{i \in I} \pi_{i} \varphi_{\lambda} \mu_{i}\left(M_{i}\right)$ for all $\lambda \in \Lambda$. For every $i \in I$ and every $\lambda \in \Lambda$, let $N_{i, \lambda}=\pi_{i} \varphi_{\lambda} \mu_{i}\left(M_{i}\right)$. Therefore,

$$
\sum_{\lambda \in \Lambda} \varphi_{\lambda}(M)=\sum_{\lambda \in \Lambda} \sum_{i \in I} \pi_{i} \varphi_{\lambda} \mu_{i}\left(M_{i}\right)=\sum_{\lambda \in \Lambda}\left(\sum_{i \in I} N_{i, \lambda}\right)=\bigoplus_{i \in I}\left(\sum_{\lambda \in \Lambda} N_{i, \lambda}\right) .
$$

Since each $M_{i}(i \in I)$ is dual Baer, each $M_{i}(i \in I)$ has the $S S S P$ by Theorem 2.23. Thus $\sum_{\lambda \in \Lambda} N_{i, \lambda}$ is a direct summand of $M_{i}$ for every $i \in I$. So $\sum_{\lambda \in \Lambda} \varphi_{\lambda}(M)$ is a direct summand of $M$. Consequently, $M$ is a dual Baer module. 
We conclude this paper by showing a new characterization of dual Baer modules.

Let $M$ be an $R$-module with $S=\operatorname{End}_{R}(M)$. Then for every nonempty subset $A$ of $S$, we denote $l_{S}(A)=\{\varphi \in S \mid \varphi A=0\}$ and $r_{M}(A)=\{m \in M \mid A m=0\}$. We also denote $l_{S}(N)=\{\varphi \in S \mid \varphi(N)=$ $0\}$ for any submodule $N$ of $M$.

Recall that a ring $R$ is called a Baer ring if for every nonempty subset $I \subseteq R$, there exists an idempotent $e \in R$ such that $l_{S}(I)=R e$.

Proposition 2.29. ([5, Proposition 2.3]) For an $R$-module $M, S=E_{n}(M)$ is a Baer ring if and only if $r_{M}\left(l_{S}\left(\sum_{\varphi \in A} \operatorname{Im} \varphi\right)\right)$ is a direct summand of $M$ for all nonempty subsets $A$ of $S$.

The next example shows that if $M$ is a module such that $S=\operatorname{End}_{R}(M)$ is a Baer ring, then $M$ is not a dual Baer module, in general.

Example 2.30. Consider the $\mathbb{Z}$-module $M=\mathbb{Z}$. Then $S=E n d_{\mathbb{Z}}(M) \cong \mathbb{Z}$. Clearly, $\mathbb{Z}$ is a Baer ring. On the other hand, it is easily seen that $M$ is not a dual Baer module.

Note that if $M$ is an $R$-module with $S=\operatorname{End}_{R}(M)$, then for any nonempty subset $A$ of $S, l_{S}(A)=$ $l_{S}(A M)$, where $A M=\sum_{f \in A} \operatorname{Im} f$. The next result can be considered as an analogue of [8, Theorem 3.5].

Theorem 2.31. The following are equivalent for an $R$-module $M$ and $S=\operatorname{End}_{R}(M)$ :

(i) $M$ is a dual Baer module;

(ii) $S$ is a Baer ring and $A M=r_{M}\left(l_{S}(A M)\right)$ for every nonempty subset $A$ of $S$;

(iii) $S$ is a Baer ring and $I M=r_{M}\left(l_{S}(I M)\right)$ for every right ideal $I$ of $S$.

Proof. (i) $\Rightarrow$ (ii) From [15, Theorem 3.6], it follows that $S$ is a Baer ring. Moreover, we have $r_{M}\left(l_{S}(A M)\right)=r_{M}\left(l_{S}(A)\right)=r_{M}(S(1-e))=e(M)=A M$ for all nonempty subsets $A$ of $S$.

(ii) $\Rightarrow$ (iii) This is obvious.

(iii) $\Rightarrow$ (i) Let $I$ be a right ideal of $S$. Since $S$ is a Baer ring, $r_{M}\left(l_{S}(I M)\right)$ is a direct summand of $M$ by Proposition 2.29. But $I M=r_{M}\left(l_{S}(I M)\right)$. Then $I M$ is a direct summand of $M$. By Theorem 2.23, it follows that $M$ is a dual Baer module.

Combining Theorem 2.31 and [10, Theorem 4.1], we get the following result.

Corollary 2.32. Let $M$ be an $R$-module such that $I M=r_{M}\left(l_{S}(I M)\right)$ for every right ideal $I$ of $S=$ $\operatorname{End}_{R}(M)$. If $M$ is a Baer module, then $M$ is a dual Baer module.

\section{References}

[1] F. W. Anderson, K. R. Fuller, Rings and Categories of Modules, vol. 13, Springer-Verlag, New York 1992.

[2] E. P. Armendariz, A note on extensions of Baer and P.P.-rings, J. Austral. Math. Soc. 18(4) (1974) 470-473.

[3] G. F. Birkenmeier, J. Y. Kim, J. K. Park, Polynomial extensions of Baer and quasi-Baer rings, J. Pure Appl. Algebra 159(1) (2001) 25-42.

[4] K. A. Byrd, Rings whose quasi-injective modules are injective, Proc. Amer. Math. Soc. 33(2) (1972) $235-240$.

[5] S. M. Khuri, Baer endomorphism rings and closure operators, Canad. J. Math. 30(5) (1978) 10701078. 
[6] I. Kaplansky, Rings of Operators, W. A. Benjamin Inc., New York-Amsterdam 1968.

[7] G. Lee, S. T. Rizvi, C. S. Roman, Rickart modules, Comm. Algebra 38(11) (2010) 4005-4027.

[8] G. Lee, S. T. Rizvi, C. S. Roman, Dual Rickart modules, Comm. Algebra 39(11) (2011) 4036-4058.

[9] S. H. Mohamed, B. J. Müller, Continuous and Discrete Modules, London Math. Soc. Lecture Notes Series 147, Cambridge University Press 1990.

[10] S. T. Rizvi, C. S. Roman, Baer and quasi-Baer modules, Comm. Algebra 32(1) (2004) 103-123.

[11] S. T. Rizvi, C. S. Roman, Baer property of modules and applications, Advances in Ring Theory (2005) 225-241.

[12] D. W. Sharpe, P. Vámos, Injective Modules, Cambridge University Press, Cambridge 1972.

[13] Y. Talebi, N. Vanaja, The torsion theory cogenerated by $M$-small modules, Comm. Algebra 30(3) (2002) 1449-1460.

[14] D. K. Tütüncü and R. Tribak, On dual Baer modules, Glasgow Math. J. 52(2) (2010) 261-269.

[15] D. K. Tütüncü, P. F. Smith, S. E. Toksoy, On dual Baer modules, Contemp. Math. 609 (2014) $173-184$.

[16] R. Wisbauer, Foundations of Module and Ring Theory, Gordon and Breach Science Publishers, Philadelphia 1991. 